\title{
New Fluorescent Alkoxy-Functionalized Perfluoroaryl Self-Assembled Low Molecular Weight Gelators
}

\author{
Tawfik A. Khattab ${ }^{1 *}$ and Maher A. El-Hashash ${ }^{2}$ \\ ${ }^{1}$ Textile Research Division, National Research Center, Cairo, Egypt \\ ${ }^{2}$ Chemistry Department, Faculty of Science, Ain Shams University, Cairo, Egypt
}

\section{A R T I C L E I N F O}

Article history:

Received 23 October 2014

Accepted 15 December 2014

Keywords:

Self-Assembly;

Organogel;

Nanofibers;

Photophysical Properties;

Thermoreversible.

\begin{abstract}
A B S T R A C T
The molecular design, synthesis and photophysical properties of a new class of thermoreversible and fluorescent low molecular weight organogels from alkoxyfunctionalized perfluoroaryls are described. Both of CuI-catalyzed decarboxylative cross-coupling and $\mathrm{S}_{\mathrm{N}} \mathrm{Ar}$ (Nucleophilic Aromatic Substitution) chemistry were employed for the preparation of those highly fluorinated gelators, in high yields and excellent purity via simple filtration, from the corresponding potassium fluorobenzoate salts and aryl iodides. A variety of fluorinated para terphenyls were prepared with a variety of para terminal alkoxy tails. The UVVis absorption and fluorescence emission spectra of those new materials display a solvatochromic and solvatofluorochromic behavior respectively. Self-assembly of these molecules via cooperative $\pi-\pi$ stacking and van der Waals interactions induced gelation of a variety of organic solvents are described. Scanning electron microscopy (SEM) and transmission electron microscopy (TEM) studies reveal the formation of fiber-like nanostructures.
\end{abstract}

\section{Introduction}

The low molecular weight organogels have an increased significance due to their thermoreversibility, chemical sensitivity, and the multiplicity of their macroscale architectures ${ }^{[1-7]}$. Organogels are commonly used in different potential applications such as biomedical, cosmetics, and foodstuffs purposes ${ }^{[8-14]}$. Liquid crystalline organogels produced via self-assembled noncovalent physical entanglements have attracted significant interests. The design of liquid crystalline organogels can demonstrate unique optoelectronic properties such as improved charge transport, fluorescence, and molecular recognition [15-17]. Additionally, highly fluorinated materials usually introduce increased surface roughness that can be employed in hydrophobic/self-cleaning surfaces ${ }^{[18-19]}$. In contrast, only limited work has been explored on liquid crystalline organogels. Surprisingly, there is no report of low molecular weight organogel based on fluorinated terphenyl derivatives although fluorinated terphenyl derivatives have played significant roles in the recent advances of optoelectronic applications. Herein we report, to the best of our knowledge, the first fluorescent liquid crystalline physical organogel based

\footnotetext{
* Corresponding author.

E-mail address: tawfekkhattab@yahoo.com
}

on a rigid para terphenyl core and flexible alkoxy side chains (see Figure 1). The design, synthesis and photophysical properties of fluorinated para terphenyl derivatives with two alkoxy terminal units are investigated for potential optoelectronic applications. A copper-catalyzed decarboxylative cross-coupling starting from the corresponding potassium perfluorobenzoate salts and aryl iodides leads to the creation of an extended, rigid, and conjugated para terphenyl core with a strong fluorescence emission. The synthetic route of alkoxy-functionalized highly fluorinated terphenyls and their liquid crystalline properties have been studied recently by our research group. Those highly fluorinated para terphenyls are found to exhibit fluorescence in the blue region of the visible spectrum and are suitable candidates for low energy consuming displays especially in the case of portable electronics ${ }^{[\mathbf{2 0 - 2 2}]}$. They also are able to function as solvatochromic and solvatofluorochromic probes. The supramolecular selfassembly and gelation properties of fluorinated para terphenyls in some organic solvents are investigated. Both of SEM and TEM are used to study the novel properties of the nano-scale structures that can be fabricated by the fluorescent organogel self-assembly. 

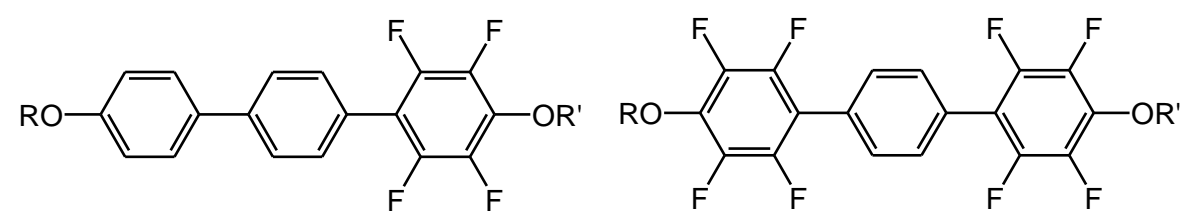

Fig. 1: Fluorinated para terphenyls comprising a rigid aromatic core and flexible alkoxy side chains; R and R' are aliphatic chains.

\section{Materials and methods}

Melting points were obtained by differential scanning calorimetry (TA instruments 2920). IR spectra were recorded with a Bruker Vectra-33 IR-Spectrometer with ATR probe. UV-Vis absorption spectra were measured on a HP-8453 (HEWLETT PACKARD) spectrophotometer. Fluorescence spectra were measured on a VARIAN CARY ECLIPSE fluorescence spectrophotometer. NMR spectra were recorded using a BRUKER AVANCE 400 spectrometer at $400 \mathrm{MHz}$; chemical shifts are given in ppm relative to internal standard TMS at $295 \mathrm{~K}$. In case of scanning electron microscopy SEM, the gel formed from 4 in $n$-propanol was scooped up and placed on carbon tapes pasted on aluminum stubs and allowed to dry at room temperature in a desiccator connected to vacuum pump. The dried samples were then annealed overnight in an oven at $45^{\circ} \mathrm{C}$, followed by application of a $10 \mathrm{~nm}$ gold-coating before recording images. SEM images were obtained using Hitachi S-2600N operating at $20 \mathrm{kV}$. For Transmission electron microscopy TEM, a piece of gel 4 in $n$-propanol was placed on a carbon-coated copper grid (200 mesh) and allowed to dry in vacuum at room temperature for two days. TEM was done on JEM$1200 \mathrm{EX}$ at an accelerating voltage of $80 \mathrm{kV}$. All materials and reagents were obtained from commercial sources and were used without further purification.

\section{Gelation procedure:}

The gelation tests were performed by dissolution of compounds 4, 5 and $\mathbf{6}$ in the selected solvents by heating to boiling temperature and then cooling to room temperature to form the gel. The gels formed in 25-45 minutes depending on the gelator concentration. Scanning electron microscopy was achieved on Hitachi S-2600N SEM (operating at $20 \mathrm{kV}$ ). The samples were prepared by drop-casting the gel in $n$-octanol on a clean glass substrates followed by drying in air to afford the xerogel. The dried xerogel was then annealed overnight in an oven at $45^{\circ} \mathrm{C}$, followed by coating with gold. For transmission electron microscopy, a piece of gel $\mathbf{4}$ in $n$ propanol was placed on a carbon-coated copper grid (200 mesh) and air-dried overnight at room temperature. TEM was done on JEM-1200EX at an accelerating voltage of $80 \mathrm{kV}$.

\section{$\mathrm{X}$-ray crystal structure information}

Single crystals of compound $\mathbf{4}$ suitable for X-ray diffraction analysis were obtained by crystallization (with slow solvent evaporation) from hexane. X-ray crystallography was performed by mounting each crystal onto a thin glass fiber from a pool of Fluorolube $^{\mathrm{TM}}$ and immediately placing it under a liquid $\mathrm{N}_{2}$ cooled $\mathrm{N}_{2}$ stream, on a Bruker AXS diffractometer. The radiation used was graphite monochromatized Mo $\mathrm{K} \alpha$ radiation $(\lambda=0.7107 \AA)$. The lattice parameters were optimized from a least-squares calculation on carefully centered reflections. Lattice determination, data collection, structure refinement, scaling, and data reduction were carried out using APEX2 version 1.0-27 software package. The structure was solved using direct methods. Subsequent Fourier synthesis yielded the remaining atom positions. The final refinement of each compound included anisotropic thermal parameters on all non-hydrogen atoms.

\section{Synthetic Procedure}

General procedure for the synthesis of a 4"alkyloxy-4-alkyloxy-2,3,5,6-tetrafluoro-1,1':4',1', terphenyl from a 4 "-alkyloxy-2,3,4,5,6-pentafluoro$1,1^{\prime}: 4$ ',1'-terphenyl

In a $100 \mathrm{ml}$ round bottom flask fitted with nitrogen bubbler and stirbar was placed 4"-alkyloxy-2,3,4,5,6pentafluoro-1,1':4',1"'-terphenyl and anhydrous solvent. The resulting mixture was heated just until the terphenyl dissolved and the respective alcohol was added. if solid came out of solution, it was heated until dissolution occurred once again. Potassium t-butoxide was added and the reaction was followed by TLC. If the reaction stalled, additional alcohol and base were added. Upon consumption of the starting material (TLC analysis) the solvent was then removed and a recrystallization was performed.

\section{4'"-propyloxy-4-hexyloxy-2,3,5,6-tetrafluoro-1-}

\section{1':4',1''-terphenyl 4}

Prepared from 4''-propyloxy-2,3,4,5,6-tetraflouro1,1':4',1' '-terphenyl (0.300 gm, $0.79 \mathrm{mmol}), n$-hexanol (5 ml, excess), DMF (15 ml), potassium $t$-butoxide (0.068 gm, $0.6 \mathrm{mmol}$ ), recrystallized from $n$-propanol, white crystals product $(0.230 \mathrm{gm}, 63 \%$ yield $) ;{ }^{1} \mathrm{H}$ NMR $\left(400 \mathrm{MHz}, \mathrm{CDCl}_{3}\right) \delta: 7.69(\mathrm{~d}, 2 \mathrm{H}), 7.59(\mathrm{~d}, 2 \mathrm{H}), 7.52$ $(\mathrm{d}, 2 \mathrm{H}), 7.02(\mathrm{~d}, 2 \mathrm{H}), 4.30(\mathrm{t}, 2 \mathrm{H}), 4.05(\mathrm{t}, 2 \mathrm{H}), 1.87(\mathrm{~m}$, $4 \mathrm{H}), 1.54(\mathrm{~m}, 2 \mathrm{H}), 1.38(\mathrm{~m}, 4 \mathrm{H}), 1.10(\mathrm{t}, 3 \mathrm{H}), 0.95(\mathrm{t}$, $3 \mathrm{H}) ;{ }^{13} \mathrm{C} \mathrm{NMR}\left(400 \mathrm{MHz}, \mathrm{CDCl}_{3}\right) \delta: 158.4,143.1$, $142.2,136.0,135.1,130.6,129.9,128.7,115.6,113.0$, 73.5, 67.3, 31.0, 29.8, 26.9, 25.0, 24.8, 18.7, 14.0, 13.9; IR (neat, $\mathrm{cm}^{-1}$ ): 3045.1, 2961.8, 2872.2, 1650.4, 1608.5; DSC $\left({ }^{\circ} \mathrm{C}\right)$ : heating-crystal $123.2 \mathrm{SmA} 144.2$ isocooling-iso 141.6 SmA 97.61 Cr. 
4'-propyloxy-4-ethoxy-2,3,5,6-tetrafluoro-1-1':4',1"'terphenyl 5

Prepared from 4"'-propyloxy-2,3,4,5,6-tetrafluoro$1,1^{\prime}: 4$ ', 1' 'terphenyl (0.300 gm, $\left.0.79 \mathrm{mmol}\right)$, ethanol (5 $\mathrm{ml}$, excess), DMF $(15 \mathrm{ml})$, potassium $t$-butoxide $(0.270$ gm, $2.4 \mathrm{mmol}$ ), recrystallized from $n$-propanol, white crystals product $(0.120 \mathrm{gm}, 37 \%$ yield $) ;{ }^{1} \mathrm{H}$ NMR (400 $\left.\mathrm{MHz}, \mathrm{CDCl}_{3}\right) \delta: 7.59(\mathrm{~d}, 2 \mathrm{H}), 7.52(\mathrm{~d}, 2 \mathrm{H}), 7.50(\mathrm{~d}, 2 \mathrm{H})$, $7.02(\mathrm{~d}, 2 \mathrm{H}), 4.37(\mathrm{q}, 2 \mathrm{H}), 4.02(\mathrm{t}, 2 \mathrm{H}), 1.86(\mathrm{~m}, 2 \mathrm{H})$, 1.49 (t, 3H), $1.10(\mathrm{t}, 3 \mathrm{H}) ;{ }^{13} \mathrm{C}$ NMR $\left(400 \mathrm{MHz}, \mathrm{CDCl}_{3}\right)$ $\delta: 157.9,144.1,141.4,136.6,134.2,129.0,128.8,128.2$, 115.2, 112.9, 72.3, 66.1, 24.8, 14.0, 12.9; IR (neat, $\mathrm{cm}^{-}$ $\left.{ }^{1}\right)$ : $3045.1,2958.2,2905.2,2865.4,1730.4$; DSC $\left({ }^{\circ} \mathrm{C}\right)$ : heating-crystal $139.3 \mathrm{~N} 147.8 \mathrm{SmA} 163.2$ iso-coolingiso $119.7 \mathrm{SmA} 101.7 \mathrm{~N} 85.86 \mathrm{Cr}$.

\section{4"'-hexyloxy-4-(2-methyl)butyloxy-2,3,5,6- \\ tetrafluoro-1,1':4',1''-terphenyl 6}

Prepared from 4"-hexyloxy-2,3,4,5,6-tetrafluoro$1,1^{\prime}: 4^{\prime}, 1$ '--terphenyl (0.210 gm, $\left.0.5 \mathrm{mmol}\right), 2$-methyl-1butanol (5 ml, excess), DMF (15 ml), potassium $t$ butoxide (0.068 gm, $0.6 \mathrm{mmol})$, recrystallized from $n$ propanol, product $(0.117 \mathrm{gm}, 48.0 \%)$; ${ }^{1} \mathrm{H}$ NMR (400 $\left.\mathrm{MHz}, \mathrm{CDCl}_{3}\right) \delta: 7.69(\mathrm{~d}, 2 \mathrm{H}, J=8.4 \mathrm{~Hz}), 7.59(\mathrm{~d}, 2 \mathrm{H}, J$ $=8.8 \mathrm{~Hz}), 7.52(\mathrm{~d}, 2 \mathrm{H}, J=8.4 \mathrm{~Hz}), 7.02(\mathrm{~d}, 2 \mathrm{H}, J=8.8$ $\mathrm{Hz}), 4.13(\mathrm{~m}, 2 \mathrm{H}), 4.04(\mathrm{t}, 2 \mathrm{H}, J=6.8 \mathrm{~Hz}), 1.88(\mathrm{~m}$, $3 \mathrm{H}), 1.63(\mathrm{~m}, 1 \mathrm{H}), 1.51(\mathrm{~m}, 2 \mathrm{H}), 1.39(\mathrm{~m}, 5 \mathrm{H}), 1.09(\mathrm{~d}$, $3 \mathrm{H}, J=6.8 \mathrm{~Hz}$ ), 0.98 (t, $3 \mathrm{H}, J=7.4 \mathrm{~Hz}), 0.93$ (t, 3H, $J$ $=6.8 \mathrm{~Hz}) \cdot{ }^{13} \mathrm{C}$ NMR $\left(400 \mathrm{MHz}, \mathrm{CDCl}_{3}\right) \delta(\mathrm{ppm})$ : $159.10,141.35,132.60,130.55,128.15,126.75,125.56$, $114.90,80.11,68.14,35.51,29.27,31.61,25.75,25.68$, $22.62,16.11,14.04,11.20 ;{ }^{19} \mathrm{~F} \quad \mathrm{NMR}(400 \mathrm{MHz}$, $\left.\mathrm{CDCl}_{3}\right) \delta(\mathrm{ppm}):-145.36$ (q, 2F), -157.51 (q, 2F); IR (neat, $v / \mathrm{cm}^{-1}$ ): 2994.0, 2862.7, 1493.2, 997.3, 801.8; DSC $\left({ }^{\circ} \mathrm{C}\right.$ )-heating-crystal $108.7 \mathrm{SmA} 125.1$ iso-coolingiso $123.7 \mathrm{SmA} 92.2$ crystal.

\section{Results and Discussion}

\section{Photophysical studies}

The UV-Vis absorption and fluorescence emission spectra of these fluorinated compounds were studied in some selected solvents. Values for $\lambda_{\max }$ were determined for compounds as listed in Table 1. The UV-Vis absorption and emission spectra for both the asymmetric series and symmetric series were measured in a variety of solvents including THF, DMSO, Pyridine, Ethanol, $\mathrm{CH}_{2} \mathrm{Cl}_{2}, \mathrm{CH}_{3} \mathrm{CN}$ and Hexane in diluted solutions to guarantee that the samples are completely soluble (see Figures 2 and 3). All derivatives were found to absorb in the UV-Vis region with maxima located below 315 $\mathrm{nm}$. Those absorption bands can be attributed to the $\pi-$ $\pi^{*}$ transitions. All derivatives display strong fluorescence in solution in the near UV-blue region of the electromagnetic spectrum. Hence, the para terphenyl derivatives display strong charge transfer character and/or a conformational relaxation in the excited state. The high radiative character of the para terphenyls can be attributed to the extended planar conjugated structural system of both core and terminal benzene rings ${ }^{[15-17]}$. On the other side, the fluorine atoms significantly enhance the intramolecular charge transfer ICT character.
The peripheral (thio)alkoxy tails, being linear or branched, only slightly influence the optical properties of para terphenyls. It was found that the increasing the length of the aliphatic chain leads to a slight increase (Bathochromic shift) in the UV-Vis absorption maxima accompanied by slight decrease (hypsochromic shift) in the fluorescence emission maxima. The asymmetric derivatives consist of organic molecular structures in which electron-donating groups (alkoxy terminal groups) are linked through a conjugated system to an electronaccepting fluorinated fragment. This kind of system, where ICT occurs, is susceptible to exhibiting solvatochromic properties. A considerable solvatochromic emission influence, known as solvatofluorochromism, was observed in different solvents with different polarities. Both UV-Vis absorption and fluorescence emission spectra display an increase in the maximal wavelength with increasing solvents polarity (positive solvatochromism). This redshift reveals that the ground state is less polar than the excited state and the fluorinated para terphenyls will be better stabilized by polar solvents.

\section{Gelation Properties}

Molecular self-assembly can be represented in different dimensions starting from the molecular level (nanometers), consisting of just a few molecules, to macroscopic level which is visible to the naked eye. Understanding the self-assembled aggregates of the organogels and liquid crystals is the main force to investigate their potential applications ${ }^{[23]}$. Organogels can be defined as a viscoelastic three dimentional solid matrix created by the entrapment of a solvent in the elastic self-assembled entanglements by either chemical or physical interactions. The strong chemical interactions affords thermally irreversible organogels whereas the physical interactions affords reversible organogels as a result of the weak non-covalent interactions such as hydrogen bonding, metal coordination, $\pi-\pi$ stacking, push-pull interactions, and van der Waals interactions ${ }^{[24]}$. The phase transition, molecular alignment and orientation control of liquid crystals are significant properties for photonic applications ${ }^{[25]}$. Organogels derived from liquid crystals comprise an entrapped mesogenic phasesegregated in a micro-scale fibrous entanglement of a low molecular weight organogel. They can function for a variety of potential technological applications such as rewritable information recording devices and electrooptical displays ${ }^{[26]}$.

The liquid crystalline highly fluorinated gelators consist of two terminal alkoxy units separated by the rigid para terphenyl core that is responsible for $\pi-\pi$ stacking interactions. The liquid crystalline properties of the gelators 4,5 and 6 have been described recently by our research group. Regarding the self-assembly of these liquid crystalline gelators, the aggregation of the fluorinated gelator molecules affords a three dimensional network of nano-scale fibrous aggregates that is controlled by the opposite forces rising from their solubility and crystals formation. Compounds 4,5 and 6 
are soluble in some selected organic solvents only at high temperature. When dissolving the powder of the fluorinated gelators 4,5 and $\mathbf{6}$ in the organic solvents by gentle heating followed by cooling the solution to room temperature, a non-flowing solid-like gel was indicated by means of the "stable to inversion" technique as shown in Figure 4. The gelation properties of compounds 4, 5 and 6 were investigated in different organic solvents under the same experimental condition. The critical gelation concentration values for liquid crystalline gelator $\mathbf{6}$ were solvent dependent and in the range of 2.34 and $7.3 \mathrm{mM}$. Gelator 6 reveals a significant ability to gelate a variety of organic solvents such as toluene $(4.2 \mathrm{mM}), n$-propanol $(2.34 \mathrm{mM}), 1,2-$ dichloroethane $(7.3 \mathrm{mM})$, and 1-octanol $(3.80 \mathrm{mM})$. However, 6 was partially gelated in tetrahydrofuran, dimethylsulfoxide and acetonitrile and showed no gelation in chloroform, ethylacetate and dichloromethane. The results of the gelation studies of compounds 4, 5 and $\mathbf{6}$ are summarized in Table 2. All gels produced from the organic solvents listed above are either white or transparent solid-like gels. The sol-gel switching process is completely thermoreversible. All investigated gelators reveal comparable photophysical properties in dilute solutions as shown in the photophysical properties.

Table 1: UV-Vis absorption and fluorescence emission parameters in some selected solvents at room temperature.

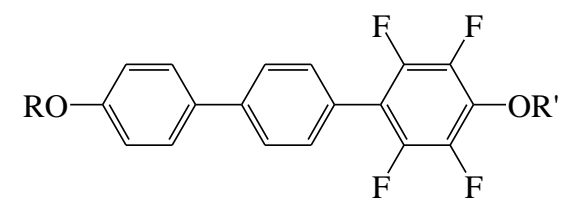

\begin{tabular}{|c|c|c|c|c|c|c|c|c|c|c|c|c|c|c|}
\hline \multirow{3}{*}{$\begin{array}{c}\text { Comp. \# } \\
\left(\mathrm{R}, \mathrm{R}^{\prime}\right)\end{array}$} & \multicolumn{14}{|c|}{$\left(\lambda_{\max } \mathrm{nm}\right)$} \\
\hline & \multicolumn{2}{|c|}{ Hexane } & \multicolumn{2}{|c|}{ DMSO } & \multicolumn{2}{|c|}{ Ethanol } & \multirow{2}{*}{$\frac{\mathrm{THF}}{a b s .}$} & \multicolumn{2}{|c|}{$\mathrm{CH}_{2} \mathrm{Cl}_{2}$} & \multicolumn{3}{|c|}{$\mathrm{CH}_{3} \mathrm{CN}$} & \multicolumn{2}{|c|}{ Pyridine } \\
\hline & $a b s$. & em. & $a b s$. & em. & abs. & em. & & em. & $a b s$. & em. & $a b s$. & em. & $a b s$. & em. \\
\hline 1 (dodecyl, hexyl) & 288 & 367 & 275 & 402 & 289 & 388 & 288 & 410 & 293 & 393 & 285 & 385 & 297 & 378 \\
\hline 2 (hexyl, hexyl) & 289 & 361 & 293 & 392 & 279 & 376 & 230 & 399 & 292 & 392 & 284 & 384 & 288 & 399 \\
\hline 3 (hexyl, ethyl) & 280 & 356 & 290 & 390 & 299 & 399 & 265 & 390 & 273 & 373 & 300 & 400 & 309 & 376 \\
\hline 4 (propyl, hexyl) & 285 & 355 & 297 & 396 & 289 & 409 & 265 & 386 & 269 & 362 & 306 & 409 & 302 & 396 \\
\hline 5 (propyl, ethyl) & 282 & 367 & 303 & 401 & 294 & 372 & 271 & 404 & 299 & 392 & 298 & 398 & 311 & 379 \\
\hline $\begin{array}{l}6 \text { (hexyl, 2- } \\
\text { methylbutyl) }\end{array}$ & 290 & 356 & 292 & 392 & 286 & 375 & 298 & 392 & 289 & 388 & 288 & 401 & 304 & 408 \\
\hline 7 (hexyl, methyl) & 288 & 356 & 293 & 398 & 286 & 376 & 292 & 399 & 289 & 375 & 294 & 387 & 305 & 385 \\
\hline
\end{tabular}

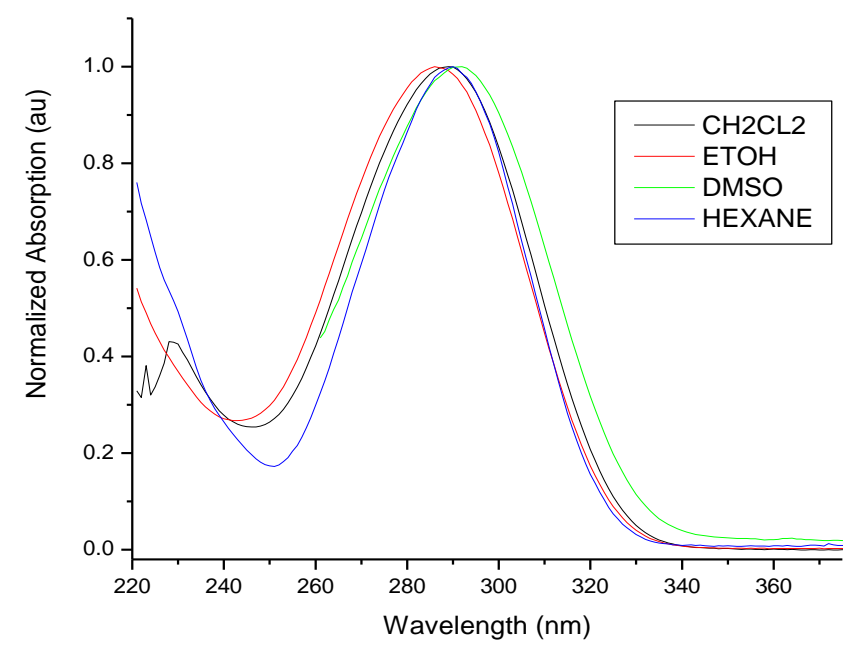

Fig. 2: Normalized UV-Vis absorption spectra of $\mathbf{6}$ in some chosen solvents. 

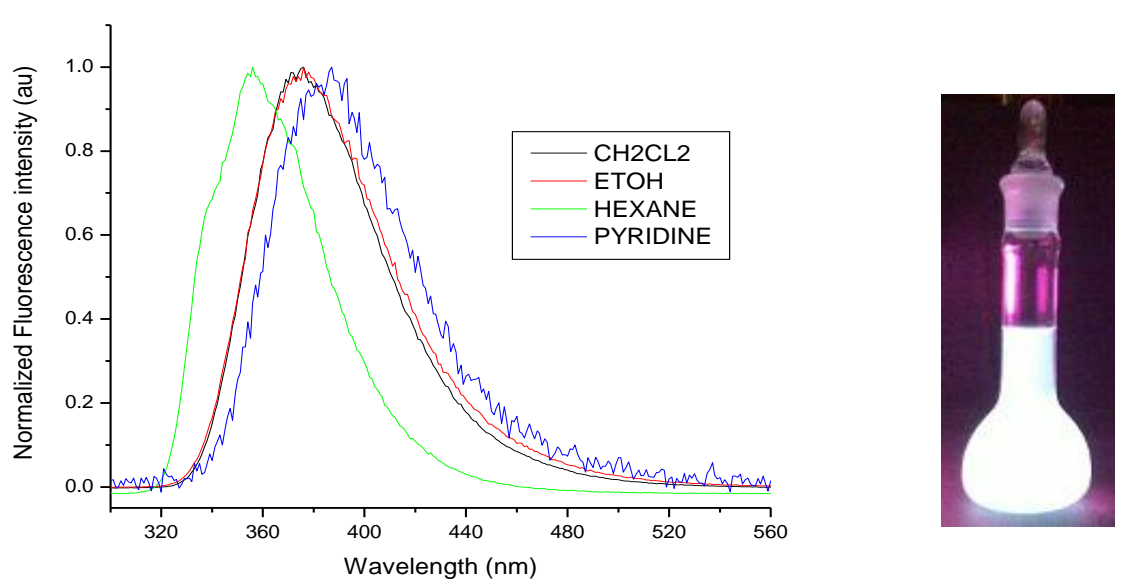

Fig. 3: Normalized fluorescence emission spectra of $\mathbf{6}$ in some chosen solvents
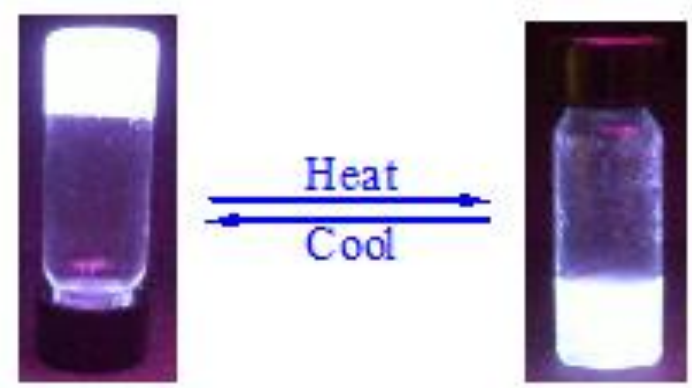

Fig. 4: Thermoreversible fluorescent organogel (using gelator 6) by means of the "stable to inversion" technique

Table 2: Gelation properties of the fluorinated gelators 4, 5 and $\mathbf{6}$ in some selected solvents.

\begin{tabular}{|l|l|l|l|}
\hline Solvent & gelator 4 & gelator 5 & gelator 6 \\
\hline$n$-Propanol & $\mathrm{G}(6.92 \mathrm{mM})$ & $\mathrm{P}$ & $\mathrm{G}(3.80 \mathrm{mM})$ \\
$n$-Octanol & $\mathrm{G}(6.55 \mathrm{mM})$ & $\mathrm{P}$ & $\mathrm{G}(2.34 \mathrm{mM})$ \\
Toluene & $\mathrm{G}(9.82 \mathrm{mM})$ & $\mathrm{P}$ & $\mathrm{G}(4.2 \mathrm{mM})$ \\
1,2-Dichloroethane & $\mathrm{PG}$ & $\mathrm{P}$ & $\mathrm{G}(7.3 \mathrm{mM})$ \\
Dichloromethane & $\mathrm{S}$ & $\mathrm{S}$ & $\mathrm{S}$ \\
Chloroform & $\mathrm{S}$ & $\mathrm{S}$ & $\mathrm{S}$ \\
Ethylacetate & $\mathrm{S}$ & $\mathrm{S}$ & $\mathrm{S}$ \\
Acetonitrile & $\mathrm{PG}$ & $\mathrm{P}$ & $\mathrm{PG}$ \\
Tetrahydrofuran & $\mathrm{PG}$ & $\mathrm{S}$ & $\mathrm{PG}$ \\
Dimethylsulfoxide & $\mathrm{PG}$ & $\mathrm{PG}$ & $\mathrm{PG}$ \\
\hline
\end{tabular}

G: gel; S: solution; PG: partial gel. The critical gelation concentration $(\mathrm{mM})$ is shown in a parenthesis.

The aggregation morphology of dried xerogel of gelator 4 was investigated by both of scanning electron microscope (SEM) and transmission electron microscope (TEM). The dried xerogel sample of liquid crystalline gelator $\mathbf{4}$ was transferred onto a glass slide and studied under SEM. Both of scanning electron microscope and transmission electron microscope images of 4 from $n$-octanol demonstrate three dimensional fibrous entanglements in the range of 320$560 \mathrm{~nm}$ in diameter and few micrometers in length (see
Figure 5). The self-assembly of $\mathbf{4}$ affords fibrous bundles leading to the immobilization of those organic solvents. The inclusion of long alkoxy units in the molecular structure of the gelators has been considered as a critical part toward better stability of the gelators assemblies. In addition to the alkoxy units included in our molecular structure gelators, the existence of fluorine atoms on the rigid core of the para terphenyls might stimulate and increase the stability of the molecular assembled architectures. To illustrate the molecular and crystal 
packing, we obtained a single crystal of compound $\mathbf{4}$ from hexane and studied under X-ray crystallography as illustrated in Figure 6. In the crystal unit, each molecule interacts with the adjacent molecules in face-to-face intermolecular $\pi$ - $\pi$ stacking interactions of the aromatic core in cooperation with tail-to-tail van der Waals interactions between the alkoxy chains. The investigation of the crystal structure of $\mathbf{4}$ suggests that molecules should have the tendency to aggregate into ladder nanostructures.

\section{Conclusions}

We have synthesized the first example of fluorescent low molecular weight organogel from fluoroterphenyl derivatives as rod-like aromatic segments that is able to immobilize a variety of organic solvents. The fluorinated para terphenyl main skeleton are prepared via practical $\mathrm{Cu}(\mathrm{I})$-assisted decarboxylative cross-coupling from their corresponding potassium perfluorobenzoate salts and aryl iodides. A convenient synthesis of fluorinated para terphenyls bearing ethers in high yields were performed by $S_{N} A r$ chemistry. The absorption and fluorescence electronic properties of these new materials display a solvatochromic and solvatofluorochromic behavior. Both of terminal alkoxy units and $\pi$ - $\pi$ stacking cooperates to form stable assembled fiber-like architectures.
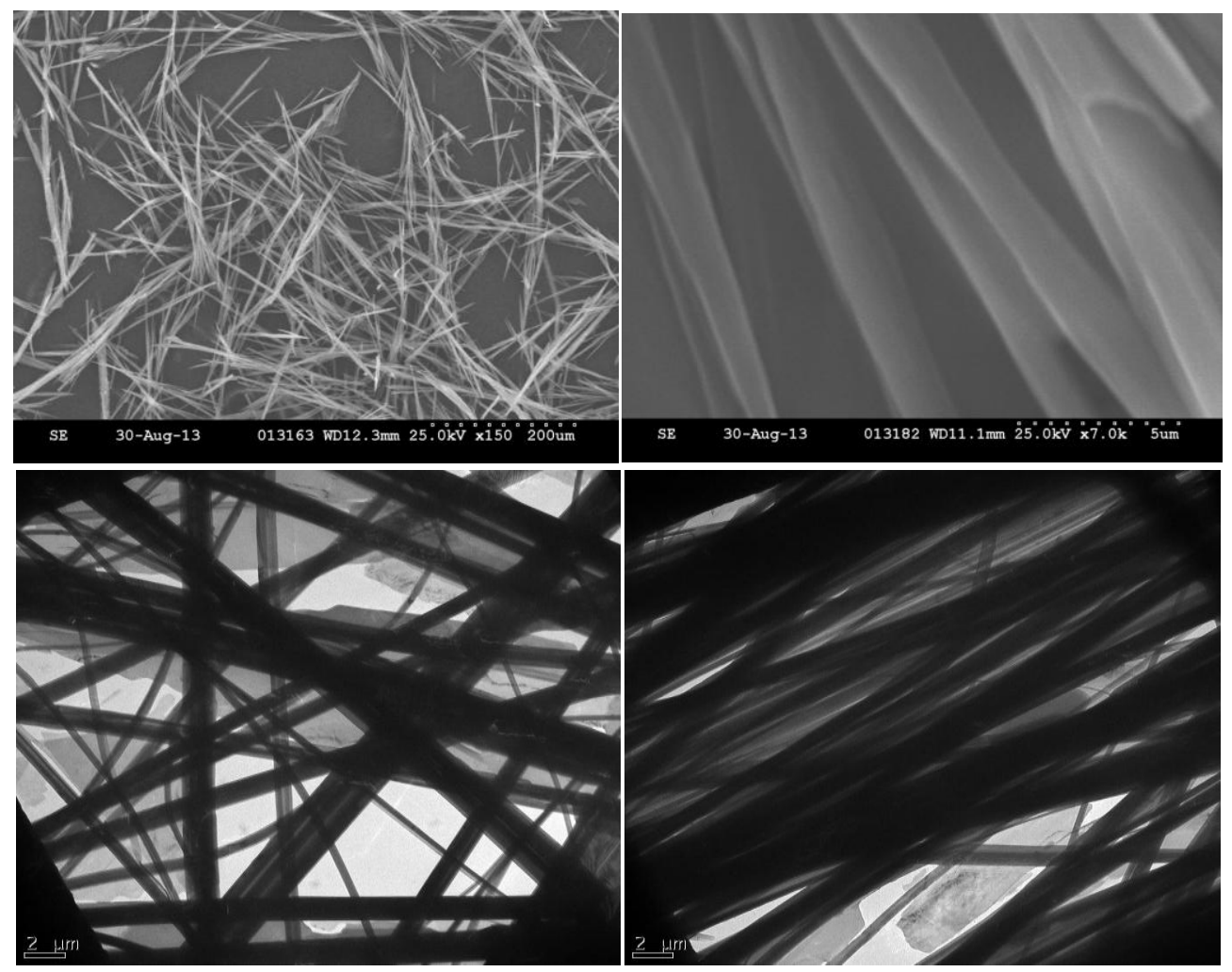

Fig. 5: (top) SEM images, and (botom) TEM images for the dried xerogel of liquid crystalline gelator 4 obtained from $n$ octanol $(6.55 \mathrm{mM})$
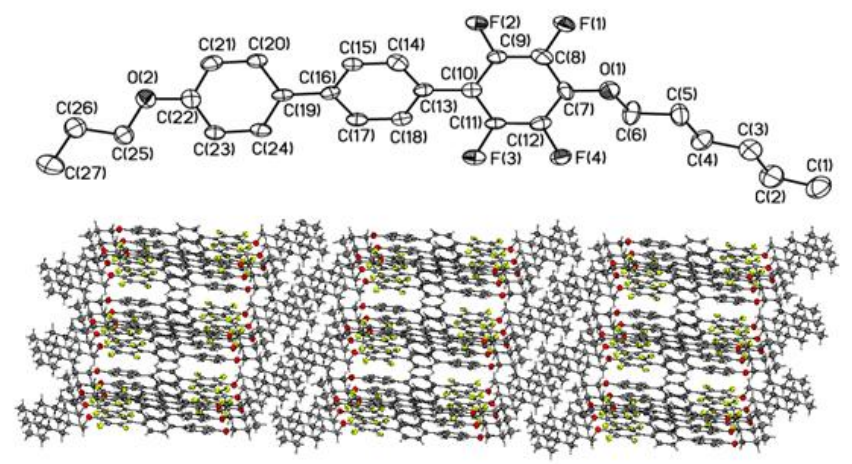

Fig. 6: (top) Crystal structure (ORTEP view) of $\mathbf{4}$ as derives from the X-ray analysis (thermal ellipsoid plots are drawn at the $30 \%$ probability level); (bottom) Face-to-face intermolecular $\pi$ - $\pi$ stacking interactions of the rigid aromatic core; and tail-to-tail van der Waals interactions between the terminal alkoxy chains 


\section{References}

1) An, B. K., Lee, D. S., Lee, J. S., Park, Y. S., Song, H. S. and Park, S. Y. (2004). Strongly fluorescent organogel system comprising fibrillar self-assembly of a trifluoromethyl-based cyanostilbene derivative. J. Am. Chem. Soc., 126(33): 10232-10233.

2) Sawada, H., Katayama, S., Ariyoshi, Y., Kawase, T., Hayakawa, Y., Tomita, T. and Baba, M. (1998). Fluorinated functional materials possessing biological activities: gel formation of novel fluoroalkylated end-capped 2-acrylamido-2methylpropanesulfonic acid polymers under noncrosslinked conditions J. Mater. Chem., 8: 15171524.

3) He, Y., Bian, Z., Kang, C., Cheng, Y. and Gao, L. (2010). Chiral binaphthylbisbipyridine-based copper (I) coordination polymer gels as supramolecular catalysts. Chemical Communications, 46(20): 35323534.

4) Asai, M., Sugiyasu, K., Fujita, N. and Shinkai, S. (2004). Facile and stable dispersion of carbon nanotubes into a hydrogel composed of a low molecular-weight gelator bearing a tautomeric dye group. Chemistry Letters, 33(2): 120-121.

5) Zhan, C., Gao, P. and Liu, M. (2005). Selfassembled helical spherical-nanotubes from an Lglutamic acid based bolaamphiphilic low molecular mass organogelator. Chemical communications, 4: 462-464.

6) Tsou, C. C. and Sun, S. S. (2006). New fluorescent amide-functionalized phenylethynylthiophene low molecular weight gelator. Organic letters, 8(3): 387390.

7) Zhang, X., Lu, R., Jia, J., Liu, X., Xue, P., Xu, D. and Zhou, H. (2010). Organogel based on $\beta$ diketone-boron difluoride without alkyl chain and H-bonding unit directed by optimally balanced $\pi-\pi$ interaction. Chemical Communications, 46(44): 8419-8421.

8) Díaz, D. D., Cid, J. J., Vazquez, P. and Torres, T. (2008). Strength enhancement of nanostructured organogels through inclusion of Phthalocyanine-containing complementary organogelator structures and in situ cross-linking by click chemistry. Chemistry-A European Journal, 14(30): 9261-9273.

9) Kim, T. H., Kim, D. G., Lee, M. and Lee, T. S. (2010). Synthesis of reversible fluorescent organogel containing 2-(2'-hydroxyphenyl) benzoxazole: fluorescence enhancement upon gelation and detecting property for nerve gas simulant. Tetrahedron, 66(9): 1667-1672.

10) Jeong, Y., Hanabusa, K., Masunaga, H., Akiba, I., Miyoshi, K., Sakurai, S. and Sakurai, K. (2005). Solvent/gelator interactions and supramolecular structure of gel fibers in cyclic bisurea/primary alcohol organogels. Langmuir, 21(2): 586-594.

11) Pal, A., Shrivastava, S. and Dey, J. (2009). Salt,
$\mathrm{pH}$ and thermoresponsive supramolecular hydrogel of N-(4-n-tetradecyloxybenzoyl)-1-carnosine. Chemical Communications, 45: 6997-6999.

12) YeuláRyu, S. and YoungáPark, S. (2004). Strong fluorescence emission induced by supramolecular assembly and gelation: luminescent organogel from nonemissive oxadiazole-based benzene-1, 3, 5tricarboxamide gelator. Chemical communications, 1: 70-71.

13) Ray, S., Das, A. K. and Banerjee, A. (2007). pHresponsive, bolaamphiphile-based smart metallohydrogels as potential dye-adsorbing agents, water purifier, and vitamin B12 carrier. Chemistry of Materials, 19(7): 1633-1639.

14) Xu, D., Liu, X., Lu, R., Xue, P., Zhang, X., Zhou, H. and Jia, J. (2011). New dendritic gelator bearing carbazole in each branching unit: selected response to fluoride ion in gel phase. Organic \& biomolecular chemistry, 9(5): 1523-1528.

15) Sato, T., Seko, M., Takasawa, R., Yoshikawa, I. and Araki, K. (2001). Mesoscopic-scale sheet-like assembly: critical role of inter-tape hydrogen bonds in the organogel formation and gel-liquid crystal transition of an alkylsilylated deoxyguanosinedodecane system. J. Mater. Chem., 11(12): 30183022.

16) Ji, Y., Luo, Y. F., Jia, X. R., Chen, E. Q., Huang, Y., Ye, C. and Wei, Y. (2005). A dendron based on natural amino acids: synthesis and behavior as an organogelator and lyotropic liquid crystal. Angewandte Chemie, 117(37): 6179-6183.

17) Ishi-i, T., Hirayama, T., Murakami, K. I., Tashiro, H., Thiemann, T., Kubo, K. and Mataka, S. (2005). Combination of an aromatic core and aromatic side chains which constitutes discotic liquid crystal and organogel supramolecular assemblies. Langmuir, 21(4): 1261-1268.

18) Jisr, R. M., Rmaile, H. H. and Schlenoff, J. B. (2005). Hydrophobic and ultrahydrophobic multilayer thin films from perfluorinated polyelectrolytes. Angewandte Chemie International Edition, 44(5): 782-785.

19) Burunkaya, E., Kiraz, N., Kesmez, Ö., Asilturk, M., Çamurlu, H. E. and Arpaç, E. (2010). Sol-gel synthesis of IPTES and D10H consisting fluorinated silane system for hydrophobic applications. Journal of sol-gel science and technology, 56(2): 99-106.

20) Chen, Z. and Swager, T. M. (2007). Synthesis and Characterization of Fluorescent Acenequinones as Dyes for Guest-Host Liquid Crystal Displays. Organic letters, 9(6): 997-1000.

21) Metrangolo, P., Prasang, C., Resnati, C., Liantonio, R., Whitwood, A. C. and Bruce, D. W. (2006). Fluorinated liquid crystals formed by halogen bonding. Chemical communications, 31: 3290-3292.

22) Bucovicean, C. M., Dong, H., Zeng, X., Pana, A., Pausescu, I., Costisor, O. and Cseh, L. (2014). Study of molecular order, mesogenic and fluorescent properties of 2, 4-bis (4-dodecyloxybenzylidene) 
cyclohexanone. Journal of Molecular Liquids, 195: 69-72.

23) Yan, X., Zhu, P. and Li, J. (2010). Self-assembly and application of diphenylalanine-based nanostructures. Chemical Society Reviews, 39(6): 1877-1890.

24) Ajayaghosh, A., Varghese, R., Mahesh, S. and Praveen, V. K. (2006). From vesicles to helical nanotubes: A Sergeant-and-Soldiers effect in the self-assembly of oligo (p-phenyleneethynylene) s. Angewandte Chemie International Edition, 45(46): 7729-7732.
25) Kamikawa, Y., Nishii, M. and Kato, T. (2004). Self-Assembly of Folic Acid Derivatives: Induction of Supramolecular Chirality by Hierarchical Chiral Structures. Chemistry-a European Journal, 10(23): 5942-5951.

26) Ariga, K., Hill, J. P., Lee, M. V., Vinu, A., Charvet, R. and Acharya, S. (2008). Challenges and breakthroughs in recent research on selfassembly. Science and Technology of Advanced Materials, 9(1): 014109. 